\title{
An Efficient Cluster-based Routing Protocol for Improvement Delay in Mobile Ad-hoc Networks
}

\author{
Marjan Najafi \\ Department of Computer Engineering, Isfahan \\ (Khorasgan) Branch, Islamic Azad University, \\ Isfahan, Iran \\ Najafi7395@gmail.com
}

\author{
MohammadReza SoltanAghaei \\ Department of Computer Engineering, Isfahan \\ (Khorasgan) Branch, Islamic Azad University, \\ Isfahan, Iran \\ Soltan@khuisf.ac.ir
}

\begin{abstract}
Cluster-based routing in Mobile Ad-Hoc Networks is considered one of the convenient methods of routing. The existence of Cluster Head $(\mathrm{CH})$ in a group of nodes for data forwarding improves the performance of routing in terms of routing overhead and power consumption. In this paper, a clustering algorithm using hierarchical routing for mobile ad hoc networks is proposed. In the proposed method, first, clusters are formed by the FLOC clustering method with low overlapping. Then, according to the most important network criteria, including hop count, noise ratio, distance, remaining energy, and link expiration time, head clusters are selected based on the weighting algorithm. To transfer data in the proposed method, two types of communication are used, e.g., inter-cluster communication and intra-cluster communication in which the shortest path to the target cluster is selected. The proposed P-METHOD technique is based on hierarchy and clustering as well as using a weighted algorithm that reduces delay in data transmission on the network. Moreover, P-METHOD is evaluated here via extensive simulations carried out in NS-2. The simulation results indicate that the MANET network performance metrics are improved in terms of average throughput, Packet Delivery Ratio (PDR), delay, residual energy, and lifetime.
\end{abstract}

Keywords- Cluster based routing; FLOC clustering method; weighting algorithm; intra-cluster communication

\section{INTRODUCTION}

Mobile ad hoc networks (MANETs) do not possess any fixed communication infrastructure. These networks consist of wireless devices that together create a self-organizing network. In mobile ad hoc networks, communications depend on intermediate nodes due to a limited transmission range of wireless interfaces; thus, each node in the network plays a router role. In these networks, the network topology is constantly changing due to the mobility of network nodes [1]. In addition, new nodes may be added to or removed from the network at any moment, or some nodes may go to sleep mode; hence, a new path should be discovered to transmit data in case of changing the topology. Since nodes in these networks continuously change their position, the need for a routing protocol that can adapt to these changes is important, so routing is one of the main challenges of these networks [2]. Regarding the continuous changes in the network topology due to the mobility of nodes, routing protocols have to support these changes by adopting a consistent strategy. One of the ways to reduce energy consumption in ad hoc networks is to use different clustering methods. In these methods, nodes are divided into distinct groups called clusters. Each cluster has a cluster head responsible for collecting information from its cluster members and transfer them to another cluster. Cluster heads play an important role in network management and control. 
Different methods are used to perform clustering which is applicable in various conditions based on the network topology and the characteristics of nodes. Therefore, in mobile ad hoc networks, prolonging the network lifetime, energy-saving, improving coverage, and load balancing are key factors in designing a clustering method.

This paper aims to design a new clustering method so that the cluster head is selected based on the most important metrics of mobile ad hoc networks including the remaining energy, distance, the received signal strength (RSS), etc. Then, the most appropriate path is selected for data transmission to reduce network latency and increase the network lifetime by reducing energy consumption.

The paper presented here is organized as the following. Section II presents the related works on cluster-based routing protocol in MANETs. In Section III, the details of our proposed P-METHOD is discussed. Moreover, parameters utilized for performance evaluation are investigated and simulation results are discussed in Section IV. Finally, in Section $\mathrm{V}$, the paper is concluded.

\section{RELATED WORKS}

There are many cluster-based routing algorithms found in literatures of MANET research. Few of such protocols related to our proposal are described in this section.

UBA method: To maximize the information collected and minimize delay, there are two central issues in the aggregation of the wireless sensor network. Most of the programs contained in the network collected are dedicated to a specific location in which total integration and delay cannot be optimized at the same time. Liu introduced a technique called UBA in 2018. This hierarchical clustering technique is proposed along with a schedule to maximize data collection. In this method, to minimize delay, information in the network is collected through a number of hubs. The two key mechanisms of UBA method are the adoption of a mixed-scheduling mode and the use of remaining energy of nodes. The best use of the remaining energy of nodes at non-focal points to implement the distribution strategy. The Time-Slot Distribution Algorithm for Children (TSDAC) of each tree has also been proposed which can help determine optimal time slot for child nodes and reduce delay performance of the network. According to simulation and accurate theoretical analysis of the proposed design, the evaluation results suggest that UBA can improve measurement information by $25 \%$ and reduce delay by $14 \%-18 \%$ over the same lifetime compared to EASDC and DAS [3].

Integrated systems and reviewing data collection: In 2018, Minhas proposed a hierarchical clustering approach for an integrated system and reviewing data collection in mines by sensor nodes. In this method, both technical and functional performance are considered in the real-world underground mine. According to previous statistics, a comprehensive set of artificial events such as node failure, falls, concentrations of gases and so on are the main factors contributing to accidents in underground mines. They have tested and validated the response and robustness of the proposed system. The system was successfully identified, and discovery of accidents in all cases are tested. The provision of a comprehensive control and monitoring mechanism and the detection location in this method was performed well. Nodes have managed to transfer data immediately by sending them to the cluster head followed by the base station. The results indicated that the system could manage to identify events successfully in all of the tested cases and to provide comprehensive control and monitoring mechanism to locate the miners and the events required for rescue operations [4].

LEACH-C improvement: The next paper, which was introduced by Khadim et al. in 2018, has addressed the improvement of the LEACH and LEACH-C protocol by using a hierarchical clustering method. This method is carried out based on two modifications: the first on the grid-balancing energy distribution by changing the amplitude of a cluster node and the other with load balancing of a number of nodes equally with mean value constant $\mathrm{N}$. The results showed that the proposed method increases network lifetime and decreases energy consumption on the network and improves the packet delivery rate [5].

Trust-based clustering: In their work, Ashwin et al. have introduced a trust-based clustering algorithm in mobile ad hoc networks. The proposed algorithm is a trust-based weighted clustering technique that investigates the effects of malicious nodes and the cluster head selection method. In the weighted clustering algorithm (WAC), all system variables are combined by weight factors based on system requirements. System variables such as the ideal node degree, communication power, mobility, and battery life of nodes are considered in this paper. Weight factors are applied based on these system requirements. The dominant set consists of a set of nodes outside the cluster head selection process, which is maintained until it fails to retrieve all nodes. This process is then documented during the system operation. Once a node is transferred from another cluster, both lists of cluster heads and their members are updated. The cluster head selection process begins with finding neighbors of each node, and the degree of difference of each node is calculated. The total distance (D_V) between each node and all its neighbors is calculated [6].

The application of GRP: In an article presented by Sudhakar et al. (2016), a three-step method was introduced to demonstrate the reduced effectiveness of the geographic routing protocol (GRP) due to the existence of malicious nodes. It builds clusters based on GRP. In this method, the cluster head is selected using a modified weighted clustering algorithm. One of the important criteria for selecting each node is node energy. More weight is given to the node with more energy. The cluster heads prevent excess overheads through a pre-determined threshold by the number of nodes supported by the cluster head. Malicious nodes are identified using a modified HELLO message, and safe keys are used to reduce the effects of breakdowns. The clustered GPR delay is much less than that of the normal GPR. As the size of the network increases, the 
end-to-end delay will decrease significantly. Cluster heads distribute public keys using fuzzy logic [7].

WBC method: The next work is an article by Sunil Pasak and his partner Sonal J., which was introduced in 2016 as WBC. Although the present study is a new work that increases the stability of clusters and reduces the missing packets, it has its own disadvantages. In this method, only two criteria (e.g., the group degree and necessary bandwidth) are considered for cluster building and cluster head selection. Important criteria such as energy, etc. are not considered. Furthermore, the routing between cluster heads is not explained [8].

PTH2 algorithm: In their paper, Milsimi et al. (2015) have proposed a priority-based protocol for a more reliable and superior selection algorithm (i.e., PTH2) to protect the confidentiality of communications from the intermediate malicious nodes. The PTH2 algorithm is a combination of a reliable priority-based selection algorithm (PTA) and priority-based headgroup selection algorithm (PHA). To detect possible paths with reliable nodes, PTA has been proposed to determine the paths without malicious nodes to forward packets based on the assigned weight with reliable parameters. In this case, PHA is also proposed based on the internal and external factors of a node to find the fastest response during the routing problem. The PTH2 algorithm aims to provide a secure algorithm and a cluster head selection algorithm for the cluster-based MANET routing protocol [9].

The comparison of the expressed approaches, along with the advantages and limitations of each method is presented in Table (1).

\section{TABLE 1. COMPARISON OF PREVIOUS METHODS}

\begin{tabular}{|c|c|c|c|c|c|}
\hline References & Algorithm Performance & $\begin{array}{c}\text { Clustering } \\
\text { Type }\end{array}$ & Table & Advantages & Disadvantages / Limitations \\
\hline [3] & $\begin{array}{l}\text { Hierarchical clustering method } \\
\text { with a scheduling program to } \\
\text { maximize data collection }\end{array}$ & $\begin{array}{l}\text { Tree } \\
\text { (hierarchical) }\end{array}$ & Yes & $\begin{array}{l}\text { Improved sensed data rate - } \\
\text { Improved delay }\end{array}$ & $\begin{array}{l}\text { Lack of flexibility in time } \\
\text { slice assignment }\end{array}$ \\
\hline [4] & $\begin{array}{l}\text { Hierarchical clustering method } \\
\text { for an integrated system and data } \\
\text { collection }\end{array}$ & Hierarchical & No & $\begin{array}{l}\text { Improved energy consumption } \\
\text { reduction and packet delivery } \\
\text { ratio }\end{array}$ & $\begin{array}{l}\text { Considering definite and } \\
\text { fixed coefficients for the } \\
\text { degree of communication } \\
\text { and intervals }\end{array}$ \\
\hline [5] & $\begin{array}{l}\text { Improved LEACH and LEACH- } \\
\text { C protocols by energy and load } \\
\text { balancing criteria }\end{array}$ & Hierarchical & No & $\begin{array}{l}\text { Improved network lifetime, } \\
\text { power consumption, and sent } \\
\text { data }\end{array}$ & \\
\hline [6] & Trust-based clustering algorithm & Hierarchical & Yes & $\begin{array}{l}\text { considering essential criteria } \\
\text { such as ideal node degree, } \\
\text { communication power, etc. }\end{array}$ & $\begin{array}{l}\text { The presence of overhead in } \\
\text { the cluster head selection due } \\
\text { to the mobility of the node to } \\
\text { select the cluster head }\end{array}$ \\
\hline [7] & $\begin{array}{l}\text { Demonstrating the reduced } \\
\text { effectiveness of the Geographic } \\
\text { Routing Protocol (GRP) due to } \\
\text { the presence of malicious nodes }\end{array}$ & Hierarchical & & $\begin{array}{l}\text { Using safe keys to reduce } \\
\text { failures - reduce overhead }\end{array}$ & \\
\hline [8] & $\begin{array}{l}\text { Considering the degree of the } \\
\text { node and bandwidth used to } \\
\text { construct the cluster and select } \\
\text { the cluster head }\end{array}$ & Hierarchical & & $\begin{array}{l}\text { Reducing the cluster head } \\
\text { changing process - cluster } \\
\text { stability and packet loss } \\
\text { reduction }\end{array}$ & Ignoring energy \\
\hline [9] & $\begin{array}{l}\text { Priority-based protocol for a } \\
\text { more reliable and superior } \\
\text { selection algorithm }\end{array}$ & $\begin{array}{l}\text { Clustering } \\
\text { based on } \\
\text { neighborhood } \\
\text { radius }\end{array}$ & No & $\begin{array}{l}\text { Considering path security - } \\
\text { selecting the path with the } \\
\text { highest response }\end{array}$ & - \\
\hline
\end{tabular}

\section{THE PROPOSED P-METHOD}

In the following section, we design a cluster-based schema by employing the hierarchical routing. The proposed system consists of three steps, such as the assumptions of the proposed method is discussed in 
Sect, A. Next section defines the proposed PMETHOD. Section C describes routing in the proposed P-METHOD.

\section{A. The assumptions of the proposed method}

The proposed method is introduced as the P-METHOD weighted clustering. In this method, the network assumptions are assumed in such a way that all nodes are considered homogeneous with similar energy. All nodes are randomly distributed. The nodes are mobile in the target region. Furthermore, the nodes have GPS by which they are aware of their location. In this project, the adopted energy model is the one used by Heintzelman et al. That is to say, considering different hypotheses about radio communication features including energy dissipation while transmitting and receiving can change the results of different methods. In the proposed method, we have considered our energy consumption model as the Heintzelman model.

This model expresses that the amount of energy a node consumes to send a k-bit message to radio transmitters in the distance greater than $\mathrm{d}$ is calculated by $E_{T X}$ and is expressed in Eq. (1).

$$
E_{T X}(k)=E_{\text {elec }} * k * E_{\text {amp }} * k * d^{2}
$$

In Eq. (1), $E_{\text {elec }}$ the dispersed radio energy, for which the value of $50 \mathrm{NJ} / \mathrm{bit}$ is allocated for the implementation of the transmitter or receiver circuit. $E_{\text {amp }}$ is the energy used for the transmission amplifier for which the value of $100 \mathrm{NJ} / \mathrm{bit}$ is allocated. In the same way, the energy dissipated by a node to receive a k-bit message that is transmitted according to the running receiver circuit $E_{\text {elec }}$ is shown by $E R x(k)$ and can be expressed by Eq. (2).

$$
\operatorname{ERx}(k)=E_{\text {elec }} * k
$$

In the proposed method, first, the nodes in the network are clustered based on the FLOC clustering method. In the FLOC method, clusters are selected with a specific radius to have the lowest overlap. This method is chosen because clusters have little overlapping; thus, the boundaries between clusters are clear. Furthermore, clusters are quickly created in this approach; therefore, this technique is used for the network structure. Each cluster has a leader called cluster head $(\mathrm{CH})$. Except for $\mathrm{CH}$, the rest of the nodes are members of the cluster. The cluster head is selected by member nodes based on the expressed weighted criteria, and the member nodes of each cluster communicate with their corresponding $\mathrm{CH}$. They do not communicate with nodes belong to other clusters. Cluster member nodes never communicate directly with cluster heads of other clusters. The cluster head of a cluster where the source node originates takes the information from the source node to the cluster head of a cluster where the destination node originates. Then, the cluster head takes the information to the destination node in its cluster. Figure 1 shows how information is sent from source to destination through the cluster heads.

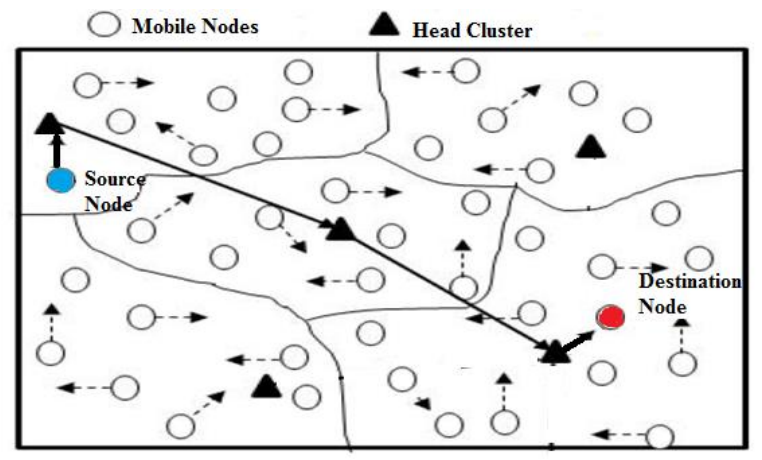

FIGURE 1 TRANSMISSION BY CLUSTERS IN THE PROPOSED METHOD

\section{B. The proposed $P$-METHOD}

The primary inefficiency of the majority of the current methods in ad hoc networks is that they are all singlepurpose. They all come with a solution, rather than a set of solutions. To select each routing method for each application, decision-makers tend to have a set of multi-purpose solutions. Our proposed P-METHOD provides a hybrid routing solution using clustering and hierarchical routing. The proposed P-METHOD is a comparative clustering protocol that uses the measured mean of five criteria, which are among the most critical system parameters in contingency networks.

The proposed P-METHOD employs several different criteria, all of which are the most critical criteria in contingency networks. The criteria used are:

Hop count: Hop count refers to the sum of the hops from a node to all its neighbors. The fewer the hops, the less energy is required for data and packet transmission, and the delay is decreased.

Signal-To-Interference-Plus-Noise Ratio (SINR): SINR is defined as the ratio of signal strength (S) to a combination of noise power $(\mathrm{N})$ and interference $(\mathrm{I})$ which can be expressed mathematically as Eq. (3).

$$
\operatorname{SINR}=\left(\frac{S}{N}+I\right)
$$

As the exact sum of the interference and noise power cannot be accurately calculated, SINR is estimated using the mean reception during the idle period. SINR is used to determine the quality of network links or connections. The higher the value of SINR, the higher the probability of a continued connection for a longer period, indicating that the node has higher signal strength.

Distance (D): The distance metric is the sum of the distance from a node to all its neighbors. If there is a long distance between a node and its neighbor, more energy and power is required to transmit data, thus increasing energy consumption. The $\mathrm{D}$ metric (i.e., the sum of distances) is obtained by Eq. (4). 


$$
\begin{aligned}
& D=\sum \operatorname{dist}(i, j) \\
& \& \\
& \text { Dist }=\sqrt{\left(x_{1}-x_{2}\right)^{2}+\left(y_{1}-y_{2}\right)^{2}}
\end{aligned}
$$

In Eq. (4), $\left(x_{1}, y_{1}\right)$ is the geographical location of the node (i) and $\left(x_{2}, y_{2}\right)$ is the geographical location of the neighboring node (j).

Residual Energy $\left(\mathrm{R}_{\mathrm{e}}\right)$ : One of the most critical factors in selecting a node as a cluster head is the amount of the remaining energy of that node. The cluster head consumes more energy than a regular node due to having more responsibilities such as key management and collected data transfer. The less energy a node consumes until it is selected as the cluster head, and the more remaining energy it has, the better it is for being chosen as the cluster head. To calculate the remaining energy, the energy consumption of each node must be calculated according to the Heintzelman model, and then the remaining energy should be calculated by Eq. (5).

$$
\begin{aligned}
& \mathrm{R}_{\mathrm{e}}(t)=P_{e}(t)-C o_{e}(t) \\
& C o_{e}(t)=E R x(k)+\mathrm{R}_{\mathrm{e}}(t)
\end{aligned}
$$

In Eq. (5):

$\mathrm{R}_{\mathrm{e}}(t)$ : The remaining energy of a node.

$P_{e}(t)$ : The initial energy of a node.

$\mathrm{Co}_{e}(t)$ : The consumed energy of a node.

$\operatorname{ERx}(k)$ : The energy consumed to receive a k-bit message

$E_{T X}$ : The energy consumed to send a k-bit message

Link Expiration Time (LET): It refers to the duration a link last. The longer this time is, the more stable the link between nodes will be. This time depends on how fast nodes move. The faster the nodes move, the more unstable the path between them, and the sooner it disappears. The link expiration time is calculated based on Eq. (6) according to packets exchanged between nodes.

$$
\begin{aligned}
& \operatorname{LET}(i, j)=\left(\frac{-(a b+c d)+\sqrt{\left(a^{2}+c^{2}\right) R^{2}-(a d-b c)^{2}}}{a^{2}+c^{2}}\right) \\
& \begin{array}{l}
a=v_{i} * \cos \theta_{i}-v_{j} * \cos \theta_{j} ; \\
b=x_{i}-x_{j} ; \\
d=Y_{i}-Y_{j} ; \\
c=v_{i} * \sin \theta_{i}-v_{j} * \sin \theta_{j} ;
\end{array}
\end{aligned}
$$

As we have stated, nodes are aware of their location using GPS. In the above equation, we have two nodes, e.g., $\mathrm{i}$ and $\mathrm{j}$, each with coordinates $\left(x_{i}, y_{i}\right)$ and $\left(x_{j}, y_{j}\right)$, $v_{i}$ and $v_{j}$ represent the movement speed of the two nodes, and $\theta_{i}, \theta_{j}$ represent the movement angle of the two nodes. All these metrics are utilized to select the cluster head and the appropriate path. The proposed PMETHOD selects the cluster head based on the weight of the nodes. However, before weighing, normalization has to be carried out as the criteria are different. For this purpose, we will follow:

$E=\left(\frac{\mathrm{R}_{\mathrm{e}, i}}{\sum_{i=1}^{N} \mathrm{R}_{\mathrm{e}}}\right):$ The remaining energy of node $i$ to the sum of the remaining energy of all cluster member nodes.

$S=\left(\frac{\operatorname{SINR}_{i}}{\sum_{i=1}^{N} \operatorname{SINR}_{i}}\right):$ The signal-to-noise ratio of node $i$ to the sum of SINR of all cluster member nodes.

$R=\left(\frac{D_{i, j}}{\sum_{i=1}^{N} D_{i, j}}\right):$ The distance between two nodes to the sum of the distances between all cluster member nodes.

$H=\left(\frac{\text { HopCount }_{i, j}}{\sum_{i=1}^{N} \text { HopCount }_{j}}\right):$ The number of hops between two nodes to the sum of the hops between all cluster member nodes.

$L=\left(\frac{L E T_{(i, j)}}{\sum_{i=1}^{N} L E T_{(i, j)}}\right):$ Link expiration time of node $i$ to node $j$

to the sum of the link expiration time between all cluster member nodes.

According to the explanations given above, the remaining energy parameters, SINR, and link expiration time are directly correlated with node selection. The more the number of these criteria are, the better the node and its associated links. However, the distance and hop count metrics are negatively correlated with node selection. The less the distance and hop count, the more appropriate the node, reducing data transmission delay. Hence, the weight assigned to node $\mathrm{X}$ is defined as Eq. (7):

$$
\begin{aligned}
& W_{X}=\sum_{i=1}^{N}\left(\begin{array}{l}
\left(W_{1} * E\right)+\left(W_{2} * S\right)+\left(W_{3} * L\right)+ \\
\left(W_{4} *(1-R)\right)+\left(W_{5} *(1-H)\right)
\end{array}\right) \\
& W_{1}+W_{2}+W_{3}+W_{4}+W_{5}=1
\end{aligned}
$$

Selecting the value for $W_{1}$ through $W_{5}$ depends on the importance of each of these metrics in our network. After calculating the weight for each node, according to the explanations given above, in each criterion, the node with the highest weight is selected. These criteria cause cluster head selection to circulate between nodes, and a particular node is not always chosen as the cluster head. This cluster head circulation balances the energy consumption of all nodes. Furthermore, considering the distance and hop count criteria will contribute to a reduction in delay. P-METHOD mainly aims to reduce 
the power consumption of all nodes and reduce network latency, which in turn increases the contingency network lifetime.

\section{Routing in the proposed P-METHOD}

The proposed P-METHOD can continuously evaluate different paths and select the best path for transmitting data to the destination. The proposed method has the following phases:

Installation Phase: This phase is when the routing table is created in each mobile node, and the cluster head is selected.

Data Transfer Phase: Data will be sent from a source to the destination when cluster heads are selected according to the estimated mean of the criteria expressed. Then, either intra-cluster or inter-cluster communication is used to transfer data.

\section{1) Installation Phase}

This phase consists of the following steps:

- Nodes arrange themselves in the form of local clusters (i.e., FLOC clustering method).

- The cluster head selection is non-intermittent and is performed whenever the network topology is changed.

- As mentioned in Section 3.3, the proposed method is based on the measured mean of the combination of different criteria.

- The cluster head selection is based on the expressed parameters and Eq. (4.7)

- For each node Xi, we evaluate the $W_{X}$ value using the following parameters, e.g., remaining energy, noise ratio, total distance, total hop count, and link expiration time.

- For each node, this information and $W_{1}$ through $W_{5}$ values are set to calculate the node weight in a table called the $X_{i}$ node routing table.

- Each node stores the values of these parameters in its routing table and updates their path regularly for optimization.

- All $X_{i}$ nodes with neighbors update their routing tables.

- Once the routing table is updated, the ith node sends its measured information only to its neighbors by Eq. (7).

- The node with the largest measured sum among all parameters will be selected as the cluster head.

- The cluster head node announces its selection by sending a message to all cluster member nodes.

\section{2) Data Transfer Phase}

The proposed P-METHOD performs both intra-cluster and inter-cluster communications for data transfer in the routing update process to avoid possible additional costs

Intra-cluster communication: If the source and destination are in a cluster, the data packet can be transmitted directly or by the cluster head. That is to say, when the destination is in the range of the source, the source and destination can communicate with each other directly or by the cluster head. Otherwise, the source and destination must exchange data through the cluster head.

Inter-cluster communication: If the source and destination are in separate clusters, the source must include an inter-cluster strategy. Simply put, first, the source sends a request message (RREQ) to the cluster head attached to it. Then, the cluster head will send the request message to its adjacent cluster heads. This process will continue until the RREQ message reaches the destination node cluster. Finally, the destination cluster head returns an RREP message to the source via the discovered path. Note that the cluster head returns the RREP message through the paths discovered via the shortest local path. Thus, the source will get the shortest path to the destination, which reduces network latency.

The proposed P-METHOD is a clustering method for contingency networks which can select the best cluster head. Then, it transmits data to the destination via a short and appropriate path. The Flowchart is illustrated in Fig. 2.

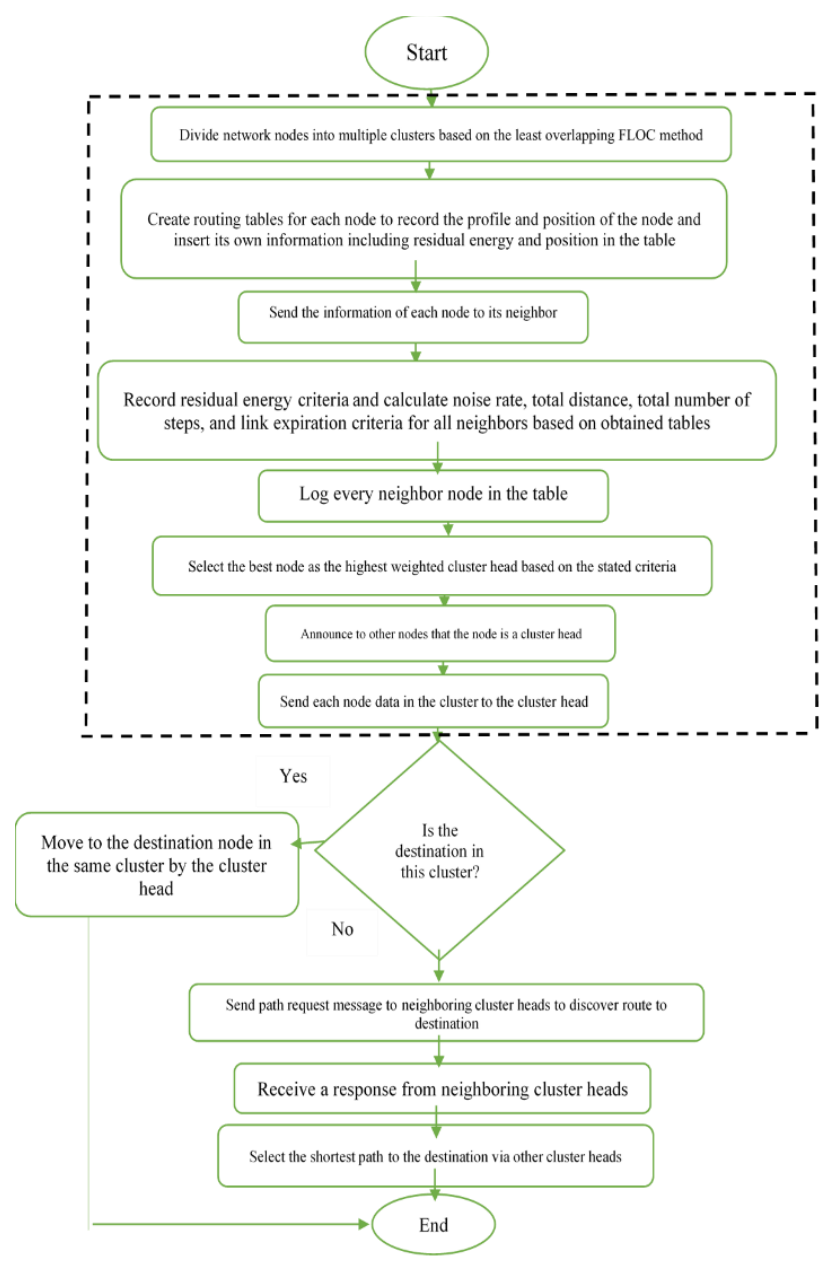

FIGURE 2 FLOWCHART OF THE PROPOSED P-METHOD

\section{EVAluating THE PERFORMANCE}

In the following section, the performance of our proposed P-METHOD approach is evaluated to location-aware distributed clustering. 


\section{A. Performance metrics}

In this section, the effectiveness and performance of our proposed P-METHOD approach is thoroughly evaluated with comprehensive simulations. The results are compared with WBC approach proposed in [8] respectively. The average throughput, Packet Delivery Ratio (PDR), end to end delay, and packet loss rate are evaluated.

\section{1) Average throughput}

Average throughput is the division of the sum of packets sizes received at the destination sensor node, to the difference of simulation stop and start time. Eq. (8) obtains the average throughput for $\mathrm{N}$ experiments, and is calculated in Kilobits per second [10].

$$
\text { Throughput }=\frac{1}{n} * \frac{\sum_{i=1}^{n} X_{i} * P_{s}}{S_{p}-S_{T}} * \frac{8}{1000}
$$

\section{2) Packet delivery ratio}

PDR is the division of the total data packets received at the destination sensor node, to the total number of data packets transmitted by the source node, described in percentage. The average PDR obtained for experiments is demonstrated by Eq. (9) [11-14].

$$
P D R=\frac{1}{n} * \frac{\sum_{i=1}^{n} X_{i}}{\sum_{i=1}^{n} Y_{i}} * 100 \%
$$

\section{3) End to end delay}

This is the average delay between the sending of packets by the source and its receipt by the receiver. This includes all possible delays caused during data acquisition, route discovery, queuing, processing at intermediate nodes, retransmission delays at the MAC, propagation time, etc. It is measured in milliseconds. The lower value of end-to-end delay means the better performance of the protocol [15-19]. The Delay is calculated in Eq. (10) follows:

$$
\text { Delay }=\left(\frac{\sum_{j=1}^{n} \text { DeliveryTime }-\sum_{j=1}^{n} \text { ArrivalTime }}{\sum_{j=1}^{n} \text { Re cievedPackets }}\right)
$$

\section{4) Residual energy}

As demonstrated in Eq. (11), unconsumed energy in the node in an arbitrary time instance is the excessive energy maintained in the node following a concluded communication with the receiver. Examples of residual energies are the energy for transmission, energy for reception, wasted energy in the system $\left(E_{s y s}\right)$, fading effects, etc. The parameters exploited for the average residual energy are listed in Table 2.:

$$
\text { Energy }_{\text {residual }}=\text { Energy }_{\text {initial }}-\left\{E T_{X}+E R_{X}+E_{\text {sys }}\right\}
$$

$$
\begin{aligned}
& E T_{X}(1 b, d i)=\left\{l b E_{\text {elec }}+l b \varepsilon_{f s} d i^{2}, d i<d i_{0}\right\} \\
& =\left\{l b E_{\text {elec }}+l b \varepsilon_{m p i} d i^{4}, d i \geq d i_{0}\right\}
\end{aligned}
$$

Energy consumed during packet transmission $E T_{X}(1 b, d i)$ and packet reception $\left(E R_{X}\right)$ are calculated using Eq. (12), and Eq. (13), respectively.

$$
E R_{X}=l b E_{\text {elec }} .
$$

If $d i>d i_{0}$, multipath fading effect occurs, and energy is wasted during transmission. However, since the fading scheme is out of the scope of this paper, the distance is considered to be lesser than the Fraunhofer's distance. Moreover, the information for channel state is not considered, while transmission efficiency is considered to be 1 .

\section{5) Lifetime}

According to the definition, the network lifetime is the elapsed time between of communication and sensing commencement with the receiver, and the time in which the final communication link from active node to the receiver is broken. Lifetime for all active nodes currently in communication with the receiver is the life time aggregate for all the mentioned nodes at any time instance. If the network is clustered, the lifetime is the total lifetime for all $\mathrm{CH}$ s. Eq. (14) demonstrated the calculation of the $N L$ value.

$$
N L=\sum_{i=1}^{m} L C H_{i}
$$

Where $\mathrm{LCH}_{i}$ is the lifetime of ith $\mathrm{CH}$.

\section{B. Simulation Setup and Comparing Algorithms}

The difficulties in implementation and debugging routing protocols in real networks, raises the necessity to consider simulations as a fundamental design tool. The main advantage of simulation is simplifying analysis and protocol verification, mainly in large-scale systems [20-33]. It is possible to employ a NAM in the NS-2 to visualize the results. In this section, the performance of our proposed approach is evaluated using NS-2 as the simulation tool, and the results are discussed further. Moreover, the proposed P-METHOD are compared with WBC model. It is worth mentioning that all P-METHOD and WBC parameters and settings are considered to be equal.

\section{Simulation results}

We have simulated P-METHOD approached in the NS2 on Linux Fedora 10. The setting of simulation parameters is given in Table 2 . 


\begin{tabular}{|c|c|}
\hline Parameters & Value \\
\hline Simulator & NS-2 (Version 2.35) \\
\hline Channel type & Wireless channel \\
\hline Radio propagation model & Propagation/Two ray ground \\
\hline Application Layer Protocol & Constant Bit Rate (CBR) \\
\hline Transport Layer Protocol & User Datagram Protocol (UDP) \\
\hline Simulation time & 300 Second \\
\hline Number of nodes & 100 \\
\hline Topographical area & $800 \times 800 \mathrm{~m}^{2}$ \\
\hline Transmission range & $150 \mathrm{~m}$ \\
\hline Mobility & Random waypoint \\
\hline MAC layer & IEEE 802.11 \\
\hline Max. node movement speed & $10 \mathrm{~m} / \mathrm{s}$ \\
\hline Pause times & $100,150,200,250,300$ second \\
\hline Packet size & 512 Bytes \\
\hline
\end{tabular}

Tables 3-7 compares the performance of $\mathrm{P}$ METHOD with that of WBC in terms of average throughput, PDR, delay, residual energy, and lifetime.

TABLE 3. AVERAGE THROUGHPUT OF TWO APPROACHES WITH VARYING DEGREE OF TIMES

\begin{tabular}{|c|c|c|}
\hline Time & P-Method & WBC \\
\hline 100 & 890.45 & 703.65 \\
\hline 150 & 940.16 & 780.65 \\
\hline 200 & 1001.54 & 795.45 \\
\hline 250 & 1165.44 & 870.54 \\
\hline 300 & 1205.44 & 890.29 \\
\hline
\end{tabular}

TABLE 4. PDR OF TWO APPROACHES WITH VARYING DEGREE OF TIMES

\begin{tabular}{|c|c|c|}
\hline Time & P-Method & WBC \\
\hline 100 & 83.91 & 57.38 \\
\hline 150 & 80.12 & 52.19 \\
\hline 200 & 78.08 & 49.98 \\
\hline 250 & 75.57 & 45.87 \\
\hline 300 & 72.88 & 37.38 \\
\hline
\end{tabular}

TABLE 5. DELAY OF TWO APPROACHES WITH VARYING DEGREE OF TIMES

\begin{tabular}{|c|c|c|}
\hline Time & P-Method & WBC \\
\hline 100 & 49 & 66 \\
\hline 150 & 53 & 75 \\
\hline 200 & 70 & 90 \\
\hline 250 & 98 & 150 \\
\hline 300 & 111 & 183 \\
\hline
\end{tabular}

TABLE 6. RESIDUAL ENERGY OF TWO APPROACHES WITH VARYING DEGREE OF TIMES

\begin{tabular}{|c|cc|}
\hline Time & P-Method & WBC \\
\hline 100 & 9.56565 & 8.5643 \\
\hline 150 & 9.343 & 7.245 \\
\hline 200 & 8.62424 & 6.0443 \\
\hline 250 & 8.0446 & 5.1347 \\
\hline 300 & 7.13535 & 5.837 \\
\hline
\end{tabular}

TABLE 7. LIFETIME OF TWO APPROACHES WITH VARYING DEGREE OF TIMES

\begin{tabular}{|c|c|c|}
\hline Time & P-Method & WBC \\
\hline 100 & 906 & 705 \\
\hline 150 & 835 & 638 \\
\hline 200 & 792 & 533 \\
\hline 250 & 718 & 406 \\
\hline 300 & 659 & 320 \\
\hline
\end{tabular}

Throughput: Figure 3, and Table 3 demonstrates the comparison between our proposed P-METHOD scheme, and NHBADI model in terms of throughput. This was predictable due to good delivery ratios and low latency. In the proposed P-METHOD, high-energy cluster heads with a short distance from cluster members are selected for data transmission. Furthermore, these cluster heads use short paths with high expiration times and lower noise ratios to send data. All these considerations have increased the throughput of the proposed method.

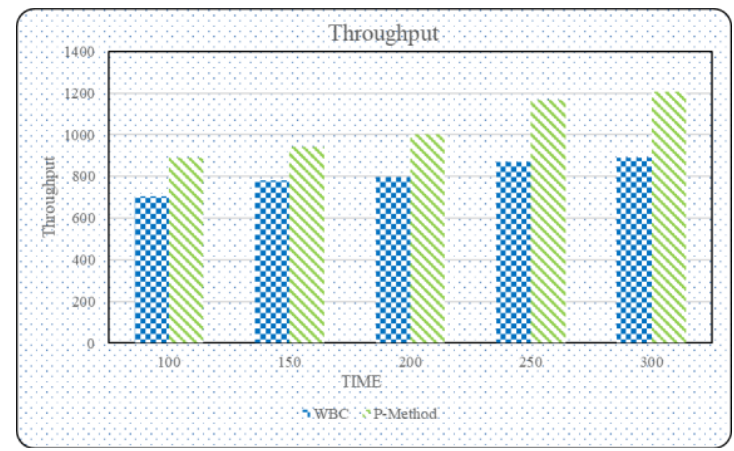

FIGURE 3 COMPARISON OF THE P-METHOD PROPOSED SCHEME AND WBC MODEL IN TERM OF AVERAGE THROUGHPUT.

PDR: As presented in Figure 4 and Table 4, The method presented in this paper (i.e., P-METHOD) has a better packet delivery ratio compared to the WBC method, which is due to packet routing using highenergy cluster heads together with paths with high expiration times. This will cause the path not to be lost during packet forwarding and the energy of the nodes not to end; thus, more packets will arrive at the destination. 


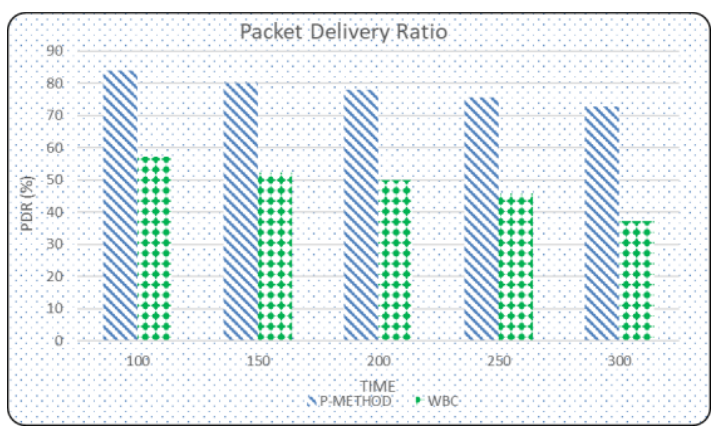

FIGURE 4 comparison of the P-METHOD proposed scheme and WBC model in term of PDR.

End-to-end delay: Figure 5, and Table 5 demonstrates the comparison between our proposed scheme, and WBC model in terms of delay. The method presented in this thesis, i.e., P-METHOD, has a lower end-to-end delay than the WBC method. This is mainly because, in the proposed method, the cluster head selection is based on the metrics of hop count and less distance. Furthermore, the shortest path is selected to send data between cluster heads. This choice causes the data to reach the destination with less delay.

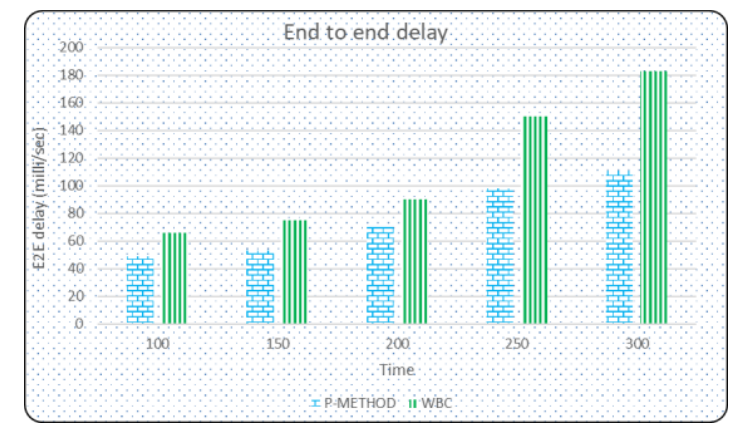

FIGURE 5 COMPARISON OF THE P-METHOD PROPOSED SCHEME AND WBC MODEL IN TERM OF DELAY.

Residual energy: As presented in Figure 6 and Table 6, the average residual energy in the nodes was performed in 20 to 100 seconds in each simulation. The average residual energy of the proposed method (P-METHOD) is higher than that of the WBC method. This is mainly because the proposed method uses those cluster heads for routing that are considered in hop count, noise ratio, distance, remaining energy, and link expiration time. Therefore, considering a low distance will reduce the energy consumption and selecting a higher energy cluster head will control the network energy and increase the remaining energy. Thus, the proposed method performs better in this case, as well. This selection in each round causes not only a certain number of nodes to be selected but the entire network to be used. Hence, in the proposed method compared to WBC, the average energy consumed is dispersed throughout the network and is reduced. According to the diagrams, the proposed P-METHOD outperforms the WBC method in terms of the remaining energy with much lower fluctuations. The energy stability in network nodes causes that we have a better network and our nodes do not die prematurely due to overuse. In the proposed P-METHOD compared to the WBC method, the amount of energy decreases over time is much lower and the remaining energy is always higher. The reason for the decline in energy is that the energy of nodes decreases over time. However, in our proposed method, as higher energy nodes are selected as the cluster head in each round, it conserves more energy compared to the WBC method.

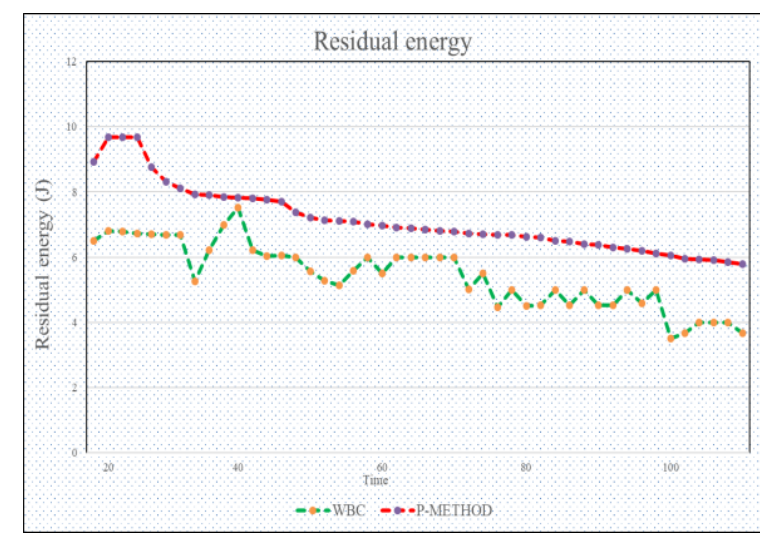

FIGURE 6 COMPARISON OF THE P-METHOD PROPOSED SCHEME AND WBC MODEL IN TERM OF AVERAGE RESIDUAL ENERGY.

Network lifetime: Figure 7, and Table 7 demonstrates the comparison between our proposed scheme, and WBC model in terms of network lifetime. P-METHOD avoids the premature death of network nodes by selecting a high-energy cluster head with a low hop count. Since cluster heads are selected based on the remaining energy and shorter distances, and lower noise with higher link expiration times are selected, the node energy is discharged over a longer period and the network lifetime is increased. In the simulation, the network lifetime decreases over time but is still higher compared to the WBC method. The reason for a reduced lifetime over time is energy consumption and the death of nodes.

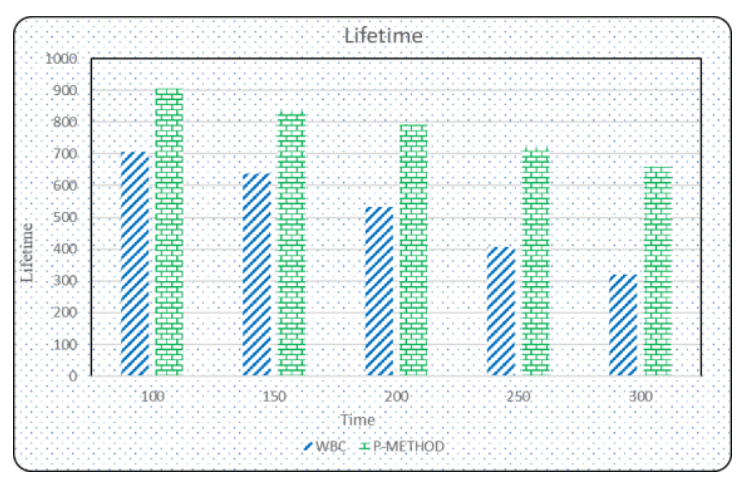

FIGURE 7 COMPARISON OF THE P-METHOD PROPOSED SCHEME AND WBC MODEL IN TERM OF LIFETIME.

\section{CONCLUSIONS}

Routing is one of the major challenges in mobile contingency networks. Due to constant changes in and movement of nodes, the network topology changes rapidly. Therefore, routing must support these changes with a consistent strategy so that the data are transmitted from source to destination correctly with the least delay. It should be conducted in such a way as 
to try to keep the network alive. This thesis presented a clustering approach for mobile contingency networks. In the proposed P-METHOD, clustering was first performed using the FLOC method, in which clusters are selected in such a way as to have the least overlap. This is a top-down hierarchical approach. Then, the cluster heads are selected based on the most important network criteria, including the remaining energy, noise ratio, total distance, total hop count, and link expiration time using the weighted method. Two types of communication have been used for data transmission in the proposed P-METHOD, e.g., intra-cluster communication and inter-cluster communication. In inter-cluster communication, the cluster head selects the shortest path to send data to the destination. The proposed P-METHOD was simulated and evaluated by the NS-2 simulator and compared with the WBC method in terms of end-to-end delay, packet delivery ratio, throughput, the average remaining energy for mobile nodes, and network lifetime.

The evaluation results show that the proposed method is more efficient than the WBC method in terms of these criteria. The simulation results of the proposed method suggested that this method can keep the network energy in a more stable state and increase the network lifetime as well as increase the packet delivery ratio and reduce latency.

\section{REFERENCES}

[1] Shafi, S., \& Ratnam, D. V. (2019). A Cross Layer Cluster Based Routing Approach for Efficient Multimedia Data Dissemination with Improved Reliability in VANETs. Wireless Personal Communications, 1-18.

[2] Robinson, Y. H., Balaji, S., \& Julie, E. G. (2019). Design of a buffer enabled ad hoc on-demand multipath distance vector routing protocol for improving throughput in mobile ad hoc networks. Wireless Personal Communications, 106(4), 20532078.

[3] Zandiyan S, Fotohi R, Koravand M. P-method: Improving AODV routing protocol for against network layer attacks in mobile Ad-Hoc networks. International Journal of Computer Science and Information Security. 2016 Jun 1;14(6):95.

[4] Jamali, S., \& Fotohi, R. (2017). DAWA: Defending against wormhole attack in MANETs by using fuzzy logic and artificial immune system. the Journal of Supercomputing, 73(12), 5173-5196.

[5] Lodeiro-Santiago, M., Caballero-Gil, P., Aguasca-Colomo, R., \& Caballero-Gil, C. (2019). Secure UAV-Based System to Detect Small Boats Using Neural Networks. Complexity, 2019.

[6] Fotohi, R., Heydari, R., \& Jamali, S. (2016). A Hybrid routing method for mobile ad-hoc networks. Journal of Advances in Computer Research, 7(3), 93-103.

[7] Fotohi, R., \& Bari, S. F. (2020). A novel countermeasure technique to protect WSN against denial-of sleep attacks using firefly and Hopfield neural network (HNN) algorithms. The Journal of Supercomputing, 1-27.

[8] Liu, Q., \& Liu, A. (2018). On the hybrid using of unicastbroadcast in wireless sensor networks. Computers \& Electrical Engineering, 71, 714-732.

[9] Sarkohaki, F., Fotohi, R., \& Ashrafian, V. (2017). An efficient routing protocol in mobile ad-hoc networks by using artificial immune system. International Journal of Advanced Computer Science and Applications (IJACSA), 8 (4).

[10] Fotohi, R., Ebazadeh, Y., \& Geshlag, M. S. (2016). A new approach for improvement security against DoS attacks in vehicular ad-hoc network. International Journal of Advanced Computer Science and Applications, 7(7), 10-16.
[11] Behzad, S., Fotohi, R., Balov, J. H., \& Rabipour, M. J. (2018). An Artificial Immune Based Approach for Detection and Isolation Misbehavior Attacks in Wireless Networks. JCP, 13(6), 705-720.

[12] Mabodi, K., Yusefi, M., Zandiyan, S., Irankhah, L., \& Fotohi, R. Multi-level trust-based intelligence schema for securing of internet of things (IoT) against security threats using cryptographic authentication. The Journal of Supercomputing, $1-25$.

[13] Fotohi, R., Jamali, S., Sarkohaki, F., \& Behzad, S. (2013). An Improvement over AODV routing protocol by limiting visited hop count. International Journal of Information Technology and Computer Science (IJITCS), 5(9), 87-93.

[14] Minhas, U. I., Naqvi, I. H., Qaisar, S., Ali, K., Shahid, S., \& Aslam, M. A. (2017). A WSN for monitoring and event reporting in underground mine environments. IEEE Systems Journal, 12(1), 485-496.

[15] Khadim, R., Maaden, A., Ennaciri, A., \& Erritali, M. (2018). An Energy-Efficient Clustering Algorithm for WSN Based on Cluster Head Selection Optimization to Prolong Network Lifetime. International Journal of Future Computer and Communication, 7(3).

[16] Ashwin, M., Kamalraj, S., \& Azath, M. (2017). Weighted clustering trust model for mobile ad hoc networks. Wireless Personal Communications, 94(4), 2203-2212.

[17] Sudhakar, S., \& Pandian, S. C. (2016). Hybrid cluster-based geographical routing protocol to mitigate malicious nodes in mobile ad hoc network. International Journal of Ad Hoc and Ubiquitous Computing, 21(4), 224-236.

[18] Pathak, S., \& Jain, S. (2016). A novel weight based clustering algorithm for routing in MANET. Wireless Networks, 22(8), 2695-2704.

[19] Fotohi, R. (2020). Securing of Unmanned Aerial Systems (UAS) against security threats using human immune system. Reliability Engineering \& System Safety, 193, 106675

[20] Fotohi, R.; Nazemi, E. An Agent-Based Self-Protective Method to Secure Communication between UAVs in Unmanned Aerial Vehicle Networks. Preprints 2020, 2020010229 (doi: 10.20944/preprints202001.0229.v1).

[21] Behzad, S., Fotohi, R., \& Jamali, S. (2013). Improvement over the OLSR routing protocol in mobile Ad Hoc networks by eliminating the unnecessary loops. International Journal of Information Technology and Computer Science (IJITCS), 5(6), 2013.

[22] Behzad, S., Fotohi, R., \& Dadgar, F. (2015). Defense against the attacks of the black hole, gray hole and wormhole in MANETs based on RTT and PFT. International Journal of Computer Science and Network Solutions (IJCSNS), 3, 89103.

[23] Seyedi, B., \& Fotohi, R. NIASHPT: a novel intelligent agentbased strategy using hello packet table (HPT) function for trust Internet of Things. The Journal of Supercomputing, 1-24.

[24] Fotohi, R., Bari, S. F., \& Yusefi, M. (2019). Securing Wireless Sensor Networks Against Denial-of-Sleep Attacks Using RSA Cryptography Algorithm and Interlock Protocol. International Journal of Communication Systems.

[25] Mylsamy, R., \& Sankaranarayanan, S. (2016). A preferencebased protocol for trust and head selection for cluster-based MANET. Wireless Personal Communications, 86(3), 16111627.

[26] Fotohi, R., \& Jamali, S. (2014). A comprehensive study on defence against wormhole attack methods in mobile Ad hoc networks. International journal of Computer Science \& Network Solutions, 2, 37-56.

[27] Jamali, S., \& Fotohi, R. (2016). Defending against wormhole attack in MANET using an artificial immune system. New Review of Information Networking, 21(2), 79-100.

[28] Jamali, S., Fotohi, R., Analoui, M. (2018). An Artificial Immune System based Method for Defense against Wormhole Attack in Mobile Adhoc Networks. TABRIZ JOURNAL OF ELECTRICAL ENGINEERING, 47(4), 1407-1419

[29] Zaminkar, M., Sarkohaki, F., \& Fotohi, R. A method based on encryption and node rating for securing the RPL protocol 
communications in the IoT ecosystem. International Journal of Communication Systems, e4693.

[30] Faraji-Biregani, M., \& Fotohi, R. (2020). Secure communication between UAVs using a method based on smart agents in unmanned aerial vehicles. The Journal of Supercomputing, 1-28.

[31] Zaminkar, M., \& Fotohi, R. (2020). SoS-RPL: Securing Internet of Things Against Sinkhole Attack Using RPL Protocol-Based Node Rating and Ranking Mechanism. WIRELESS PERSONAL COMMUNICATIONS.

[32] Midya, S., Roy, A., Majumder, K., \& Phadikar, S. (2020). QoS aware distributed dynamic channel allocation for $\mathrm{V} 2 \mathrm{~V}$ communication in TVWS spectrum. Computer Networks, 171, 107126.

[33] Gyawali, S., Xu, S., Qian, Y., \& Hu, R. Q. (2020). Challenges and Solutions for Cellular based V2X Communications. IEEE Communications Surveys \& Tutorials. 